\title{
Dielectric response and tunability of a dielectric-paraelectric composite
}

K. Zhou, S. A. Boggs, R. Ramprasad, M. Aindow, C. Erkey, and S. P. Alpay'

Citation: Appl. Phys. Lett. 93, 102908 (2008); doi: 10.1063/1.2982086

View online: http://dx.doi.org/10.1063/1.2982086

View Table of Contents: http://aip.scitation.org/toc/apl/93/10

Published by the American Institute of Physics

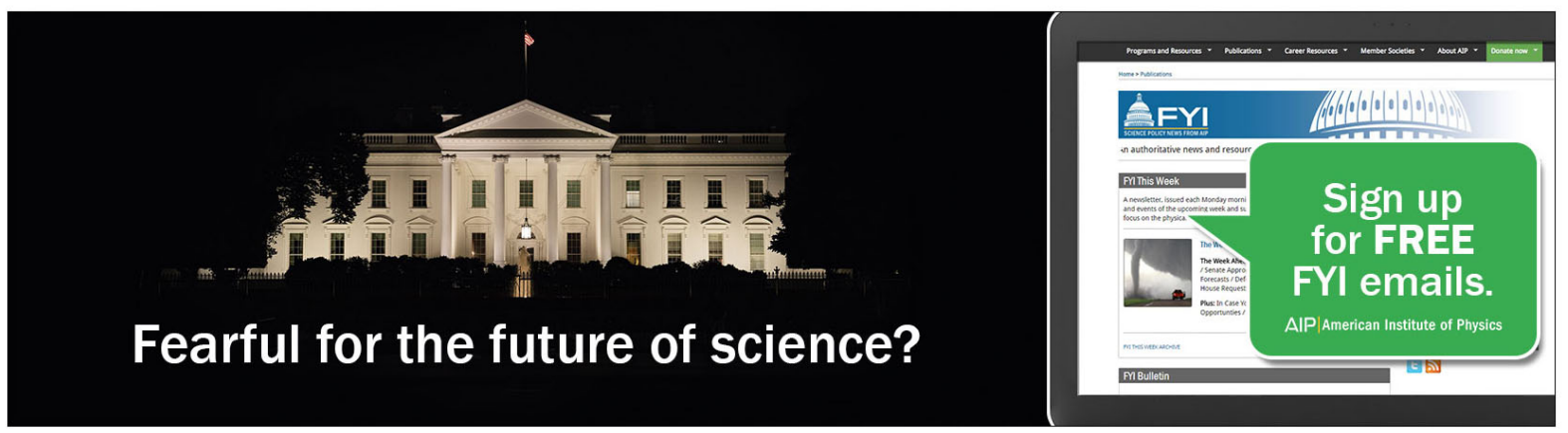




\title{
Dielectric response and tunability of a dielectric-paraelectric composite
}

\author{
K. Zhou, ${ }^{1}$ S. A. Boggs, ${ }^{1}$ R. Ramprasad, ${ }^{1,2}$ M. Aindow, ${ }^{1,2}$ C. Erkey, ${ }^{3}$ and S. P. Alpay ${ }^{1,2, a)}$ \\ ${ }^{1}$ Institute of Material Science, University of Connecticut, Connecticut 06269, USA \\ ${ }^{2}$ Materials Science and Engineering Program, Department of Chemical, Materials, and Biomolecular \\ Engineering, University of Connecticut, Connecticut 06269, USA \\ ${ }^{3}$ Department of Chemical and Biological Engineering, Koç University, 34450 Sartyer, Istanbul, Turkey
}

(Received 22 July 2008; accepted 16 August 2008; published online 12 September 2008)

\begin{abstract}
A theoretical study was carried out to determine the dielectric response and tunability of a composite consisting of a linear, low-loss dielectric matrix with uniformly sized, randomly distributed paraelectric $\mathrm{Ba}_{0.60} \mathrm{Sr}_{0.40} \mathrm{TiO}_{3}$ (BST 60/40) particles as functions of the volume fraction and size of the particles. The field dependence of the polarization and the dielectric response of the inclusions are specified through a nonlinear thermodynamic model and then incorporated into a two-dimensional finite element analysis. Near the percolation threshold for BST particles ( $\sim 27 \%$ to $45 \%$ depending on the particle size), high dielectric tunabilities with a lower effective permittivity than monolithic BST can be realized. (C) 2008 American Institute of Physics.
\end{abstract}

American Institute of Physics [DOI: 10.1063/1.2982086]

Due to their highly nonlinear dielectric response to an applied electric field, ferroelectric (FE) materials have attracted significant interest as active elements of tunable microwave devices for wireless telecommunications, including tunable mixers, delay lines, filters, and phase shifters for steerable antennas. ${ }^{1-3}$ Barium strontium titanate $\left(\mathrm{Ba}_{x} \mathrm{Sr}_{1-x} \mathrm{TiO}_{3}\right.$ or $\left.\mathrm{BST}\right)$ is one of the most promising materials system for such devices owing to its high dielectric response and its tunability near the ferroelectric phase transformation temperature $T_{C}$. For bulk BST 60/40 $\left(\mathrm{Ba}_{0.60} \mathrm{Sr}_{0.40} \mathrm{TiO}_{3}\right), T_{C}$ is below room temperature $\left(-30{ }^{\circ} \mathrm{C}\right)$ and this can be controlled by adjusting the composition. Ideally, BST is employed in its paraelectric (PE) state to reduce losses associated with the hysteresis due to polarization switching. Several compositions of BST thin films have been studied in great detail for use in tunable microwave devices using a variety of different fabrication methods and substrates (see Ref. 3 for a recent review). Typically, the dielectric losses and tunability are proportional to the dielectric permittivity. ${ }^{2}$ Recent reports indicate that high dielectric tunability and relatively low losses accompanied by a minimal dispersion in dielectric properties over a large temperature interval can be achieved in FE heterostructures and graded multilayers. $^{4}$

One way of further reducing the loss tangent is by creating a composite material consisting of a low-loss linear dielectric and a nonlinear dielectric material, preferably a FE in its PE state. The determination of the dielectric properties of composite materials is a classical problem in electromagnetism that has been investigated using the effective medium theory (EMT). ${ }^{5}$ Recently, Tagantsev and co-workers ${ }^{6,7}$ have used a modified EMT that takes into account the nonlinear dielectric response of the FE material in a FE-dielectric composite. The dielectric response, and its electric field dependence, of the FE is provided through a Landau potential that is then coupled with the EMT to establish electrostatic interactions between the FE matrix and the dielectric filler. The

\footnotetext{
${ }^{\text {a) }}$ Author to whom correspondence should be addressed. Electronic mail: p.alpay@ims.uconn.edu.
}

analysis shows that addition of a small amount of linear dielectric to a FE matrix actually improves the dielectric tunability but the loss tangent of the composite remains unaffected. In this report, we present the results of a theoretical study wherein we analyze the dielectric response of a composite consisting of a linear, low-loss dielectric matrix with uniformly sized, randomly distributed PE BST 60/40 particles as functions of the fraction and size of the particles. Unlike the approach adopted in Refs. 6 and 7, the FE in its PE state is dispersed in a linear dielectric material matrix. The field dependence of the polarization and the dielectric response of these inclusions are specified through a nonlinear thermodynamic model using the Landau theory of phase transformations and then integrated into a two-dimensional finite element analysis via the commercially available COMSOL MULTIPHYSICSTM package. The results show that once the percolation threshold for BST particles at $\sim 27$ to $45 \mathrm{vol} \%$ (depending on the particle size) is reached, the dielectric tunability increases drastically with increasing volume fraction of BST.

A schematic depicting the two-dimensional model is shown in Fig. 1(a). The system is a composite thin film of dimensions $L$ and $L^{\prime}$ consisting of randomly distributed PE BST $60 / 40$ circular particles of diameter $D$ embedded in a linear dielectric matrix with a relative dielectric permittivity of 25 sandwiched between metallic electrodes. Three different particle sizes were analyzed with $D=20,50$, and $80 \mathrm{~nm}$. To obtain a better statistical variation and to minimize the standard deviation, the cross-sectional area defined by $L \times L^{\prime}$ was varied to keep the number of particles in the model approximately constant. For 20,50 , and $80 \mathrm{~nm}$ particles, $L \times L^{\prime}$ were $250 \times 500, \quad 250 \times 1250$, and 250 $\times 2500 \mathrm{~nm}^{2}$, respectively.

We first start off with the description of the electric field dependence of the dielectric response for PE BST 60/40. BST 60/40 has the prototypical cubic perovskite lattice in its PE state above $T_{C}=-30{ }^{\circ} \mathrm{C}$ and transforms to a tetragonal FE phase below this temperature. The phase transformation characteristics can be described via a Landau potential given by 

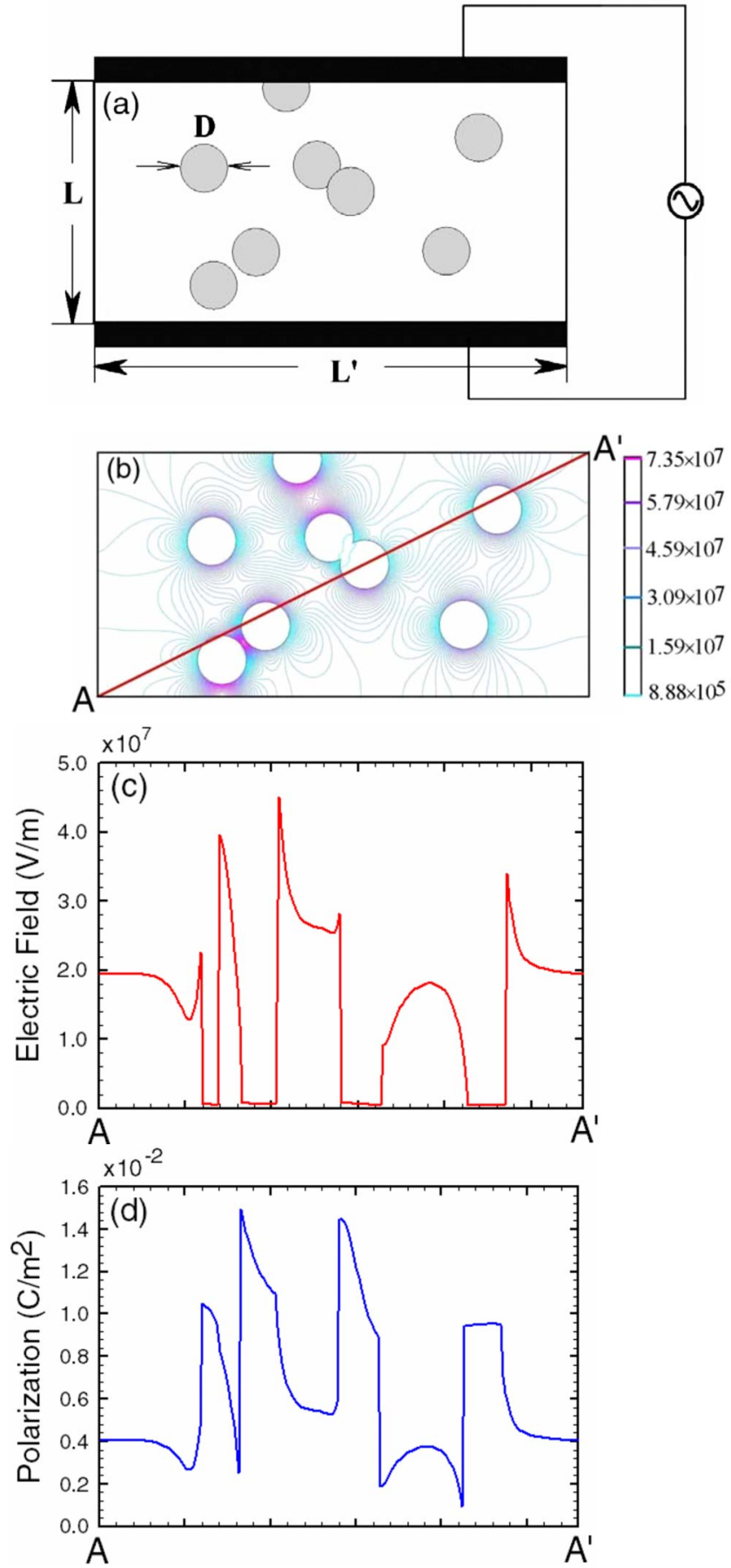

FIG. 1. (Color online) (a) Schematic of a composite material comprised of BST particles in a linear dielectric matrix, (b) electric field distribution within the composite shown in (a), the scale is in $\mathrm{V} / \mathrm{m}$, (c) electric field distribution, and (d) electric polarization distribution along section $\mathrm{AA}^{\prime}$ in (b).

$$
F(P, T, E)=F_{0}+\frac{1}{2} a P^{2}+\frac{1}{4} b P^{4}+\frac{1}{6} c P^{6}-E P,
$$

where $P$ is the polarization, $T$ is the temperature, $E$ is the applied electric field, $F_{0}$ is the energy in the PE state, and $a$, $b$, and $c$ are the dielectric stiffness coefficients. The temperature dependence of $a$ is given by the Curie-Weiss law $a=\left(T-T_{C}\right) / \varepsilon_{0} C$ where $C$ is the Curie-Weiss constant and $\varepsilon_{0}$ is the permittivity of free space. We shall assume that the BST inclusions are embedded in a compliant linear dielectric matrix and are stress free. The electric field dependence of the polarization follows from the equation of state such that

$$
\frac{\partial F(P, T, E)}{\partial P}=0=a P_{0}+b P_{0}^{3}+c P_{0}^{5}-E,
$$

where $P_{0}(E)$ is the equilibrium polarization at a given field $E$. The relative dielectric response parallel to the applied electric field and its tunability are given by

$$
\begin{aligned}
& \varepsilon_{r}(E)=\left[\varepsilon_{0} \frac{\partial^{2} F(P, T, E)}{\partial P^{2}}\right]^{-1}=\frac{1}{\varepsilon_{0}\left(a+3 b P_{0}^{2}+5 c P_{0}^{2}\right)}, \\
& \eta(E)=\frac{\varepsilon_{r}(E=0)-\varepsilon_{r}(E)}{\varepsilon_{r}(E=0)} .
\end{aligned}
$$

For BST 60/40, the relevant Curie temperature, the CurieWeiss constant, and the higher order Landau dielectric coefficients in SI units are $T_{C}=-30{ }^{\circ} \mathrm{C}, C=1.34 \times 10^{5}, b=8.64$ $\times 10^{6} \times(T-175)+3.36 \times 10^{9}$, and $c=2.38 \times 10^{9}$ (compiled from Refs. 8 and 9]. Using Eqs. (2) and (3), the dielectric response and its electric field dependence can be calculated. At RT $\left(=25^{\circ} \mathrm{C}\right)$, this results in the well known bell-shaped curve typical for a FE material in its PE state (not shown).

For numerical modeling we use the nonlinear, time dependent Laplace equation realized in the COMSOL MULTIPHYSICS $^{\mathrm{TM}}$ finite element toolbox. To construct the composite material, randomly positioned circles of diameter $D$ representing BST particles are inserted into a rectangular dielectric matrix of dimensions $L$ and $L^{\prime}$. To calculate the effective dielectric response for the composite material, the built-in electrostatic module of COMSOL is employed that utilizes a two-dimensional Laplace relation given by $\nabla\left[\varepsilon_{0} \varepsilon_{r} \nabla V(x, y)\right]=0$ to determine the local potential $V(x, y)$ within a single mesh.

The model is first run for the geometry as illustrated in Fig. 1(a). Direct time dependent solver in the COMSOL package is then used to obtain a transient analysis, with tested time steps and tolerances. Figure 1(b) shows the electric field contour plot for an applied electric field $E$ and a corresponding potential $V$. Due to the abrupt change of dielectric response at the boundary between the BST particles and the matrix, the electric field and polarization vary greatly. In Fig. 1(c), the electric field is plotted along a section denoted $\mathrm{AA}^{\prime}$. From the straightforward application of the relevant Maxwell equations and the boundary condition corresponding to the continuity of the dielectric displacement at the dielectric/PE interface, one can conclude that due to the large difference between the dielectric response of the particles and the matrix, the electric field inside BST particles will be much lower than outside. The same result is demonstrated in Fig. 1(d) where the polarization profile along $\mathrm{AA}^{\prime}$ is plotted.

To examine the effect of the size of the PE particles on the overall dielectric response and tunability, we simulated three series of composites with particle diameters $D=20,50$, and $80 \mathrm{~nm}$. The effective small-signal dielectric response is calculated from the total energy of the system, i.e., the numerical integration of the local dielectric displacements and electrical fields. Figure 2 shows the result of composite relative dielectric response as a function of BST volume fraction and particle size. When the volume fraction is below the percolation threshold, all three of these composites show a linear dielectric response; above the percolation threshold, the response increases sharply and nonlinearly in each case. The percolation limits for the composites with 20,50, and 80 $\mathrm{nm}$ particles were determined from Fig. 2 to be $45 \%, 37 \%$, 


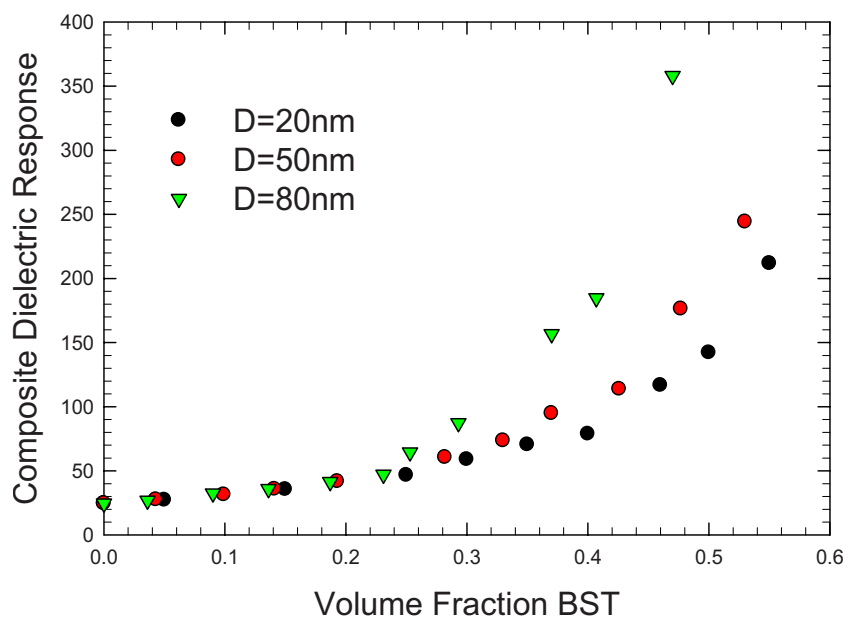

FIG. 2. (Color online) Change of the composite small-signal dielectric response as a function of BST volume fraction for different BST particle sizes.

and $27 \%$ BST, respectively. These findings are in excellent agreement with theoretical results based on Monte Carlo methods for hard spherical and semipenetrating particles. ${ }^{10,11}$ When the particles are small, the probability for them to overlap is smaller than larger particles and as such, composites with larger particles have a smaller percolation threshold, as shown in Fig. 2. Furthermore, for the same BST volume fraction, composites with larger particles have a higher dielectric response as it is easier to form a percolation path with larger particles. These results are also consistent with the findings of Jylhä and Sihvola. ${ }^{12}$ Using a similar approach wherein a Bruggeman EMT is employed together with a nonlinear thermodynamic model for the incipient FE $\mathrm{SrTiO}_{3}$ (STO) based on Vendik and Zubko, ${ }^{13}$ the dielectric tunability of a composite consisting of STO particles embedded in a linear dielectric matrix was simulated. The theoretical model shows that once the percolation limit for STO particles is reached at around $30 \mathrm{vol} \%$, the dielectric tunability increases continuously with increasing volume fraction of STO.

In Fig. 3, dielectric tunability is calculated as a function of volume fraction and particle size between zero electric field and $12 \mathrm{kV} / \mathrm{mm}$. It shows, as expected, a similar trend to the dielectric response shown in Fig. 2. Below the percolation thresholds the tunabilities for the three series of composites are very small. As the volume fraction is increased, the tunabilities increase exponentially and composites with larger BST particles have a larger tunability at the same volume fraction. We use the model of $D=80 \mathrm{~nm}$ to compare the tunability between monolithic unclamped, stress-free BST, and the composite material. At a volume fraction of $45 \%$ $\mathrm{BST}$, the relative small-signal dielectric response of the composite is 360 and the tunability is about $58 \%$ at an applied field of $12 \mathrm{kV} / \mathrm{mm}$. However, for monolithic BST, the relative dielectric response is 2300 and tunability is around $70 \%$ at $12 \mathrm{kV} / \mathrm{mm}$. This means that by employing a composite material such as the one described in this analysis, one can reduce the effective permittivity greatly while still maintain-

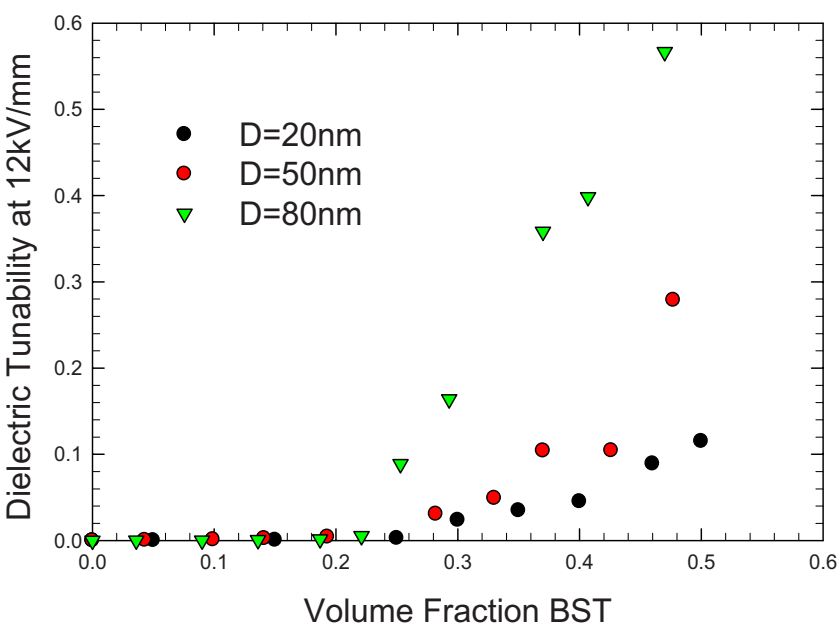

FIG. 3. (Color online) Change of dielectric tunability at $12 \mathrm{kV} / \mathrm{mm}$ as a function of BST volume fraction for different BST particle sizes.

ing a very high tunability. Finally, we note that chemical interactions between BST particles and the matrix, and their modification of the polarizability at the interfaces were not taken into account explicitly in this analysis. Surface modifications of the dielectric response can be treated separately using $a b$ initio methods, as has been done before, ${ }^{14}$ and can be included in the current treatment. Such efforts are underway.

S.P.A. gratefully acknowledges the support of the U.S. Army Research Office through Grant No. W911NF-05-10528.

${ }^{1}$ O. G. Vendik, E. K. Hollmann, A. B. Kozyrev, and A. M. Prudan, J. Supercond. 12, 325 (1999).

${ }^{2}$ A. K. Tagantsev, V. O. Sherman, K. F. Astafiev, J. Venkatesh, and N. Setter, J. Electroceram. 11, 5 (2003).

${ }^{3}$ P. Bao, T. J. Jackson, X. Wang, and M. J. Lancester, J. Phys. D 41, 063001 (2008).

${ }^{4}$ See, e.g., S. Zhong, S. P. Alpay, M. W. Cole, E. Ngo, S. Hirsch, and J. D. Demaree, Appl. Phys. Lett. 90, 092901 (2007); W. Fu, H. Wang, L. Cao, and Y. Zhou, ibid. 92, 182910 (2008).

${ }^{5}$ See, e.g., J. E. Sipe and R. W. Boyd, Phys. Rev. A 46, 1614 (1992); K. W. Yu, P. M. Hui, and D. Stroud, Phys. Rev. B 47, 14150 (1993); T. C. Choy, Effective Medium Theory: Principles and Applications (Oxford University Press, Oxford, 1999).

${ }^{6}$ K. F. Astafiev, V. O. Sherman, A. K. Tagantsev, and N. Setter, J. Eur. Ceram. Soc. 23, 2381 (2003).

${ }^{7}$ V. O. Sherman, A. K. Tagantsev, and N. Setter, J. Appl. Phys. 99, 074104 (2006).

${ }^{8}$ N. A. Pertsev, A. G. Zembilgotov, and A. K. Tagantsev, Phys. Rev. Lett. 80, 1988 (1998); N. A. Pertsev, A. K. Tagantsev, and N. Setter, Phys. Rev. B 61, R825 (2000).

${ }^{9}$ Y. L. Li, S. Choudhury, J. H. Haeni, M. D. Biegalski, A. Vasudevarao, A. Sharan, H. Z. Ma, J. Levy, V. Gopalan, S. Trolier-McKinstry, D. G. Schlom, Q. X. Jia, and L. Q. Chen, Phys. Rev. B 73, 184112 (2006).

${ }^{10}$ M. Rottereau, J. C. Gimela, T. Nicolai, and D. Durand, Eur. Phys. J. E 11, 61 (2003).

${ }^{11}$ L. An, S. A. Boggs, and J. P. Calame, Proceedings of the 2008 IEEE Power Modulator Conference (unpublished).

${ }^{12}$ L. Jylhä and A. H. Sihvola, Prog. Electromagn. Res. 78, 189 (2008).

${ }^{13}$ O. G. Vendik and S. P. Zubko, J. Appl. Phys. 82, 4475 (1997).

${ }^{14}$ N. Shi and R. Ramprasad, Phys. Rev. B 74, 045318 (2006). 